\title{
Golgi phosphoprotein 3 expression predicts poor prognosis in patients with prostate cancer undergoing radical prostatectomy
}

\author{
LONGYANG ZHANG ${ }^{*}$, FENG GUO* ${ }^{*}$ XINGHUA GAO and YANLIN WU \\ Department of Urology, Jinan Central Hospital of Shandong University, Jinan, Shangdong 250013, P.R. China
}

Received June 4, 2014; Accepted February 10, 2015

DOI: $10.3892 / \mathrm{mmr} .2015 .3455$

\begin{abstract}
Golgi phosphoprotein 3 (GOLPH3) has recently been implicated as an oncogene involved in the development of carcinoma in a number of organs. The expression of GOLPH3 in prostate cancer $(\mathrm{PCa})$ tissues was investigated in the present study. Human PC-3 and LNCaP PCa cell lines were analyzed in order to assess whether silencing of GOLPH3 expression affects cell vitality, migration and invasion, in vitro. An immunohistochemistry analysis was performed in order to measure the expression of GOLPH3 in samples from 117 patients with $\mathrm{PCa}$ and from 50 patients with benign prostatic hyperplasia (BPH). Associations between GOLPH3 expression and clinicopathological parameters, such as overall survival, were assessed. GOLPH3 expression was shown to be significantly greater in PCa tissues than in BPH tissues. GOLPH3 expression was positively correlated with Gleason score $(\mathrm{P}=0.031)$, tumor stage ( $\mathrm{T}$ stage; $\mathrm{P}=0.020$ ) and lymph node status $(\mathrm{P}=0.013)$, in patients with $\mathrm{PCa}$. Biochemical recurrence-free survival (serum prostate-specific antigen-based) and overall survival, were reduced in patients with GOLPH3-positive PCa. A multivariate analysis indicated that GOLPH3 expression was an independent predictor of biochemical recurrence-free survival [hazard ratio (HR), 2.943; 95\% confidence interval (CI), 1.190-5.521; $\mathrm{P}=0.028$ ], and of overall survival (HR, 4.371; 95\% CI, 2.045-7.109; $\mathrm{P}=0.014)$. Transfection with GOLPH3-targeted small interfering RNA reduced the capability of PC-3 and $\mathrm{LNCaP}$ cell lines to proliferate, migrate and invade in vitro, compared with the controls. The level of GOLPH3 expression in radical prostatectomy samples may be useful for predicting biochemical recurrence-free survival and overall survival in patients with PCa.
\end{abstract}

Correspondence to: Dr Feng Guo, Department of Urology, Jinan Central Hospital of Shandong University, 105 Jiefang Road, Lixia, Jinan 250013, P.R. China

E-mail: guofengdoc@163.com

*Contributed equally

Key words: golgi phosphoprotein 3, prostate cancer, prognosis

\section{Introduction}

Each year, approximately 230,000 American males are diagnosed with prostate cancer (PCa) and nearly 30,000 die from this disease $(1,2)$. However, for the majority of patients, the disease is detected at the local or regional stages, meaning that long-term prognosis is typically good (3). Radical prostatectomy is the selected treatment for $50 \%$ of patients with $\mathrm{PCa}$, of which approximately $40 \%$ exhibit aggressive clinicopathological features, such as a high Gleason score, invasion of the seminal vesicles or lymph node involvement. These features are associated with an increased risk of metastatic disease (4-6). Approximately $15 \%$ of patients with PCa are at risk of death, many receive potentially unnecessary additional postoperative interventions, such as adjuvant radiation (7). Therefore, they often experience treatment-associated morbidity (8). Furthermore, PCa-associated mortality has been observed in patients who do not exhibit adverse clinical features. Current methods for predicting the risk of metastasis and mortality in patients with $\mathrm{PCa}$ are insufficient (9). Therefore, specific genetic markers are required in order to develop prognostic indicators for patients with PCa.

Golgi phosphoprotein 3 (GOLPH3), a member of the trans-golgi matrix family, has recently been demonstrated to act as an oncogene in carcinoma of the lung, ovary, breast, colon and prostate, and in melanoma, rhabdomyosarcoma, and glioma (10-13). GOLPH3 overexpression has been reported to promote cell proliferation and tumorigenesis via activation of mammalian target of rapamycin signaling, which enhances protein kinase $\mathrm{B}$ activity and decreases transcriptional activity of the forkhead box protein $\mathrm{O}$ gene $(10,14,15)$. However, to the best of our knowledge, few studies have investigated the association between GOLPH3 expression and the survival of patients with PCa. In the present study, immunohistochemical staining of $\mathrm{PCa}$ and control prostate tissues was performed, in order to evaluate the expression of GOLPH3, and to analyze the association between GOLPH3 expression and clinicopathological factors in patients with PCa.

\section{Materials and methods}

Patients and prostate specimens. PCa samples (117) were obtained from patients with an average age of 62.4 years and a range of 48-77 years between 1999 and 2012 at the Department of Urology, Jinan Central Hospital of Shandong 
University (Jinan, China). Patients had undergone radical prostatectomy between January 1999-2012. The control resections were obtained from patients with benign prostatic hyperplasia (BPH), comprising 50 age-matched patients examined during the same period. Samples were resected from areas of invasive adenocarcinoma, which had been pathologically identified according to a hematoxylin and eosin staining pattern. Tumor grade and clinical stage of the samples were assessed according to the 2002 TNM classification and the Gleason system (16).

Follow-up. Serum prostate-specific antigen (PSA) levels were evaluated postoperatively every three months during the first year, every six months from the second to the fifth year, and then annually from the sixth year. Follow-up data were obtained by consulting medical records held by the hospital and the departmental database of patients with $\mathrm{PCa}$, and by contacting the patients or their family members. Biochemical recurrence was defined as a sustained elevation of the total serum PSA level $(>0.2 \mathrm{ng} / \mathrm{ml})$ on $\geq 2$ occasions. The biochemical recurrence date was recorded as the time that the first value was $>0.2 \mathrm{ng} / \mathrm{ml}$. Follow-up time ranged from 6 to 171 months. Patients provided informed consent. The present study was approved by the Jinan Central Hospital of Shandong University Ethical Committee (Jinan, China).

Immunohistochemistry analysis. Tissue sections $(5 \mu \mathrm{m})$ were deparaffinized, hydrated and incubated in water with $3 \% \mathrm{H}_{2} \mathrm{O}_{2}$ (Sinopharm Chemical Reagent Co., Ltd., Beijing, China) for $30 \mathrm{~min}$ in order to destroy endogenous peroxidases. Antigen retrieval was performed by immersing sections in $10 \mathrm{mM}$ citrate buffer (pH 6.0; Sinopharm Chemical Reagent Co., Ltd.) and heating in a microwave for $30 \mathrm{~min}$ at $95^{\circ} \mathrm{C}$. Non-specific binding to sections was blocked with normal goat serum [5\% (Jackson ImmunoResearch Labs, Inc., West Grove, PA, USA) in phosphate-buffered saline (Sinopharm Chemical Reagent Co., Ltd.)] for $1 \mathrm{~h}$. Subsequently, the cells were incubated with a polyclonal rabbit GOLPH3 antibody (1:100; ab91492; Abcam, Cambridge, MA, USA) overnight, at $4^{\circ} \mathrm{C}$. For the negative control, 5\% normal goat serum without a primary antibody was used. Staining was detected using a polyclonal secondary horseradish peroxidase-conjugated rabbit IgG antibody (1:2,000; ab6721; Abcam) followed by hematoxylin counter-staining. Positive staining was defined as brown oxidized 3,3'-diaminobenzidine in cellular compartments, without background signal. The stained sections were evaluated by two pathologists, unaware of patient clinical information, using light microscopy (SZ51; Olympus, Tokyo, Japan). Immunostaining scores for GOLPH3 were determined using a numeric intensity score of 0-3: 0 , no staining; $1+$, weak staining; $2+$, moderate staining and $3+$, strong staining. Staining was also dichotomized into negative and positive. Negative was scored if 0 or $1+$ and positive was scored if $2+$ or $3+$.

Cell culture and small interfering RNA (siRNA) transfection. PC-3 and LNCaP human PCa cell lines were purchased from the American Type Culture Collection (ATCC; Manassas, VA, USA). Cells were cultured in an RPMI1640 medium, supplemented with $10 \%$ fetal bovine serum (HyClone Laboratories, Inc., Logan, UT, USA) in $5 \% \mathrm{CO}_{2}$, at $37^{\circ} \mathrm{C}$.
GOLPH3-siRNA and non-specific control siRNA (Invitrogen Life Technologies, Carlsbad, CA, USA) were transfected into PC-3 and LNCaP cells using Lipofectamine $2000^{\circledR}$ (Invitrogen Life Technologies) according to the manufacturer's instructions.

Western blot analysis. Total protein was extracted from cultured cells. Equal amounts of protein $(60 \mu \mathrm{g})$ were subjected to electrophoresis using a 10\% SDS-polyacrylamide gel (Jackson ImmunoResearch Labs, Inc.) then transferred to polyvinylidene difluoride membranes (EMD Millipore, Billerica, MA, USA). Non-specific binding to membranes was blocked using $5 \%$ non-fat milk prior to incubation with polyclonal rabbit anti-GOLPH3 (1:1,000; ab91492; CA, Abcam) or monoclonal rabbit anti-GAPDH antibodies (1:2,000; ab181602; Abcam) overnight, at $4^{\circ} \mathrm{C}$. The membranes were washed and incubated with specific peroxidase-conjugated secondary antibodies. Specific proteins were detected using an enhanced chemiluminescence system (Thermo Fisher Scientific, Inc., Waltham, MA, USA).

Cell vitality, migration and invasion assays. The effect of transfection on cell growth was determined by seeding 4,000 cells/well into a 96-well plate and counting cell numbers 5 days later using 3-(4,5-dimethylthiazol-2-yl)-2,5-diphenyltetrazolium bromide reagent (MTT; Sigma-Aldrich, St. Louis, MO, USA), according to the manufacturer's instructions. The cell migratory and invasive capability levels were measured using Transwell assays. Transfected cells $\left(1 \times 10^{4}\right.$; in fetal bovine serum-free medium) were plated in the upper chambers of a Transwell plate (Corning Life Sciences, Union City, CA, USA). Lower chambers were filled with a medium supplemented with $10 \%$ fetal bovine serum. Cell invasion assays were performed similar to the cell growth assays. However, the Transwell membranes were coated with Matrigel ${ }^{\circledR}$ (Sinopharm Chemical Reagent Co., Ltd.), prior to adding the cells. At $24 \mathrm{~h}$, cells were removed from the upper chambers by swabbing, and those that had moved into the lower chambers were fixed with $4 \%$ paraformaldehyde and then stained with $0.1 \%$ crystal violet (both from Sinopharm Chemical Reagent Co., Ltd.). The number of cells in five independent fields of view for each well was recorded and photographed.

Statistical analysis. A $\chi^{2}$ test was performed in order to analyze the association between GOLPH3 expression and clinicopathological features of the patients with PCa. Survival data were evaluated using the Kaplan-Meier method and the log-rank test. Clinical parameters were analyzed using univariate and multivariate Cox proportional hazards models in SPSS version 16.0 (SPSS, Inc., Chicago, IL, USA). P<0.05 was considered to indicate a statistically significant difference.

\section{Results}

Associations between GOLPH3 expression and clinical parameters in patients with $P C a$. GOLPH3 protein expression was assessed immunohistochemically in 117 tissues from patients with $\mathrm{PCa}$ and in $50 \mathrm{BPH}$ tissues from age-matched controls. As shown in Table I, 54 of the $117 \mathrm{PCa}$ tissues (56\%) were classified as GOLPH3-positive (Fig. 1). 
Table I. Clinicopathological features of prostate cancer patients according to GOLPH3 status.

\begin{tabular}{|c|c|c|c|}
\hline Features & GOLPH3 positive & GOLPH3 negative & P-value \\
\hline Patient (n) & 54 & 63 & \\
\hline Serum PSA level (ng/ml) & & & 0.779 \\
\hline$<10$ & 25 & 29 & \\
\hline$\geq 10$ & 29 & 34 & \\
\hline Gleason score & & & 0.031 \\
\hline$\leq 6$ & 19 & 38 & \\
\hline$\geq 7$ & 35 & 25 & \\
\hline T stage & & & 0.020 \\
\hline $\mathrm{T} 1-\mathrm{T} 2$ & 35 & 54 & \\
\hline T3-T4 & 19 & 9 & \\
\hline Lymph node status & & & 0.013 \\
\hline$(-)$ & 33 & 53 & \\
\hline$(+)$ & 21 & 10 & \\
\hline Surgical margin status & & & 0.812 \\
\hline$(-)$ & 35 & 43 & \\
\hline$(+)$ & 19 & 20 & \\
\hline
\end{tabular}

GOLPH3, Golgi phosphoprotein 3; T stage, tumor stage; PSA, prostate-specific antigen.
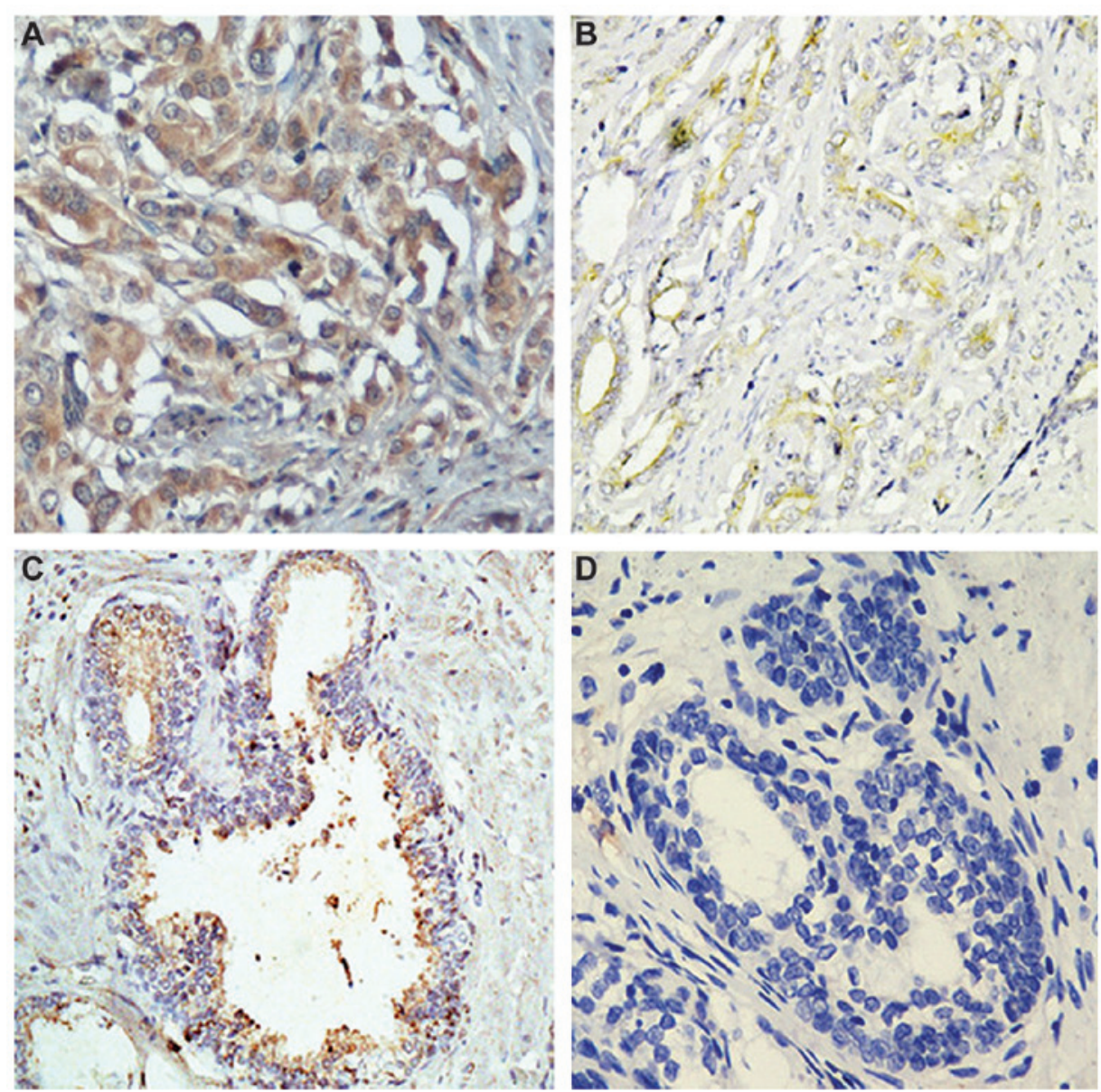

Figure 1. Immunohistochemical staining for GOLPH3 in prostate cancer (PCa) and benign prostate tissue (x200). (A) Score of 3+ for GOLPH3 expression in PCa tissues. (B) Score of 2+ GOLPH3 expression in PCa tissues. (C) Score of 1+ GOLPH3 staining in benign prostate tissue. (D) Score of 0 staining of negative controls, with the primary antibody omitted in PCa tissues. The staining intensity and pattern was graded on a 4-tier system; grade 0, negative staining; grade 1, weak staining; grade 2, moderate staining; grade 3, strong staining with membranous accentuation. Grades 2 and 3 were considered positive and grades 0 and 1 were considered negative. PCa, prostrate cancer; GOLPH3, golgi phosphoprotein 3. 
Table II. Correlations of clinical variables and GOLPH3 expression with biochemical recurrence-free survival.

\begin{tabular}{|c|c|c|c|c|}
\hline \multirow[b]{2}{*}{ Variable } & \multicolumn{2}{|c|}{ Univariate analysis } & \multicolumn{2}{|c|}{ Multivariate analysis } \\
\hline & $\operatorname{HR}(95 \% \mathrm{CI})$ & P-value & $\operatorname{HR}(95 \% \mathrm{CI})$ & P-value \\
\hline \multicolumn{5}{|c|}{ Preoperative PSA } \\
\hline$<10$ vs. $\geq 10$ & $0.696(0.462-1.189)$ & 0.496 & & \\
\hline \multicolumn{5}{|l|}{ Gleason score } \\
\hline$\leq 6$ vs. $\geq 7$ & $1.917(1.014-3.512)$ & 0.021 & $1.037(0.521-1.689)$ & 0.219 \\
\hline \multicolumn{5}{|l|}{ T stage } \\
\hline$\leq 2$ vs. $\geq 3$ & $2.192(1.334-4.328)$ & 0.019 & $1.663(0.752-2.436)$ & 0.097 \\
\hline \multicolumn{5}{|c|}{ Lymph node status } \\
\hline + vs. - & $1.029(0.570-1.968)$ & 0.837 & & \\
\hline \multicolumn{5}{|c|}{ Surgical margin status } \\
\hline + vs. - & $1.251(0.706-2.429)$ & 0.296 & & \\
\hline \multicolumn{5}{|c|}{ GOLPH3 expression } \\
\hline + vs. - & $4.257(1.985-7.235)$ & $<0.001$ & $2.943(1.190-5.521)$ & 0.028 \\
\hline
\end{tabular}

Table III. Correlations of clinical variables and GOLPH3 expression with overall survival.

\begin{tabular}{|c|c|c|c|c|}
\hline \multirow[b]{2}{*}{ Variable } & \multicolumn{2}{|c|}{ Univariate analysis } & \multicolumn{2}{|c|}{ Multivariate analysis } \\
\hline & HR $(95 \% \mathrm{CI})$ & P-value & HR $(95 \% \mathrm{CI})$ & P-value \\
\hline \multicolumn{5}{|c|}{ Preoperative PSA } \\
\hline$<10$ vs. $\geq 10$ & $0.814(0.486-2.121)$ & 0.642 & & \\
\hline \multicolumn{5}{|l|}{ Gleason score } \\
\hline$\leq 6$ vs. $\geq 7$ & $2.702(1.209-4.910)$ & 0.025 & $1.207(0.603-2.917)$ & 0.389 \\
\hline \multicolumn{5}{|l|}{ T stage } \\
\hline$\leq 2$ vs. $\geq 3$ & $2.154(1.002-3.506)$ & 0.041 & $1.497(0.477-2.098)$ & 0.745 \\
\hline \multicolumn{5}{|c|}{ Lymph node status } \\
\hline + vs. - & $1.019(0.414-2.708)$ & 0.401 & & \\
\hline \multicolumn{5}{|c|}{ Surgical margin status } \\
\hline + vs. - & $1.544(0.673-3.097)$ & 0.120 & & \\
\hline \multicolumn{5}{|c|}{ GOLPH3 expression } \\
\hline + vs. - & $4.598(2.042-12.109)$ & 0.001 & $4.371(2.045-7.109)$ & 0.014 \\
\hline
\end{tabular}

Analyses of associations between GOLPH3 expression and clinical and prognostic parameters, shown in Table I, demonstrated that GOLPH3 expression was positively correlated with Gleason score $(\mathrm{P}=0.031)$, $\mathrm{T}$ stage $(\mathrm{P}=0.020)$ and lymph node status $(\mathrm{P}=0.013)$.

Association between GOLPH3 expression and patient survival. Results of the Kaplan-Meier and log rank tests suggested that GOLPH3-positive patients exhibited a shorter biochemical recurrence-free survival time compared with GOLPH3-negative patients (Fig. 2A). GOLPH3-positive patients also demonstrated significantly shorter overall survival rates (Fig. 2B).

Univariate and multivariate analyses suggested that positive GOLPH3 expression was significantly correlated with poorer biochemical recurrence-free survival [hazard ratio (HR), 2.943; 95\% confidence interval (CI), 1.190-5.521; $\mathrm{P}=0.028$, Table II], and overall survival (HR, 4.371; 95\% CI, 2.045-7.109; $\mathrm{P}=0.014$, Table III). These results suggested that GOLPH3 expression may be useful as a prognostic indicator for patients with $\mathrm{PCa}$. 
A

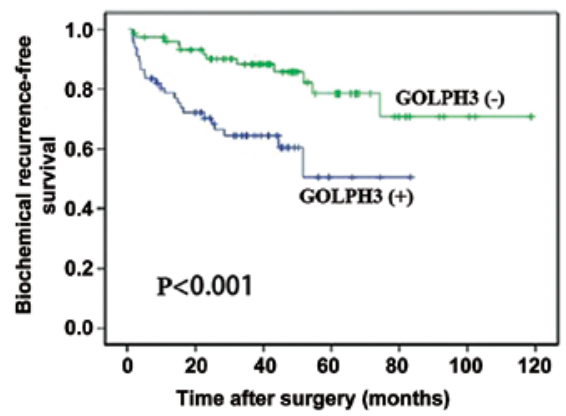

B

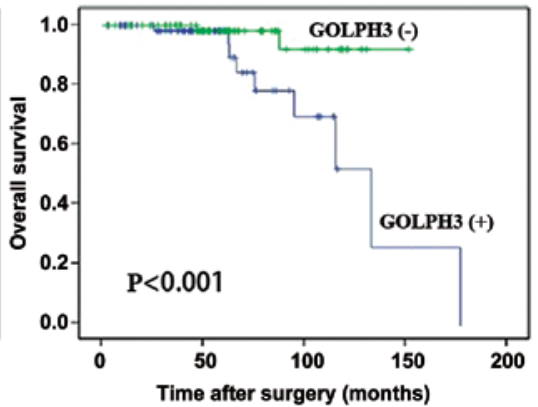

Figure 2. Prognostic value of GOLPH3 expression in prostate cancer. (A) Kaplan-Meier curves of GOLPH3 expression in biochemical recurrence-free survival and (B) overall survival. GOLPH3, Golgi phosphoprotein 3.
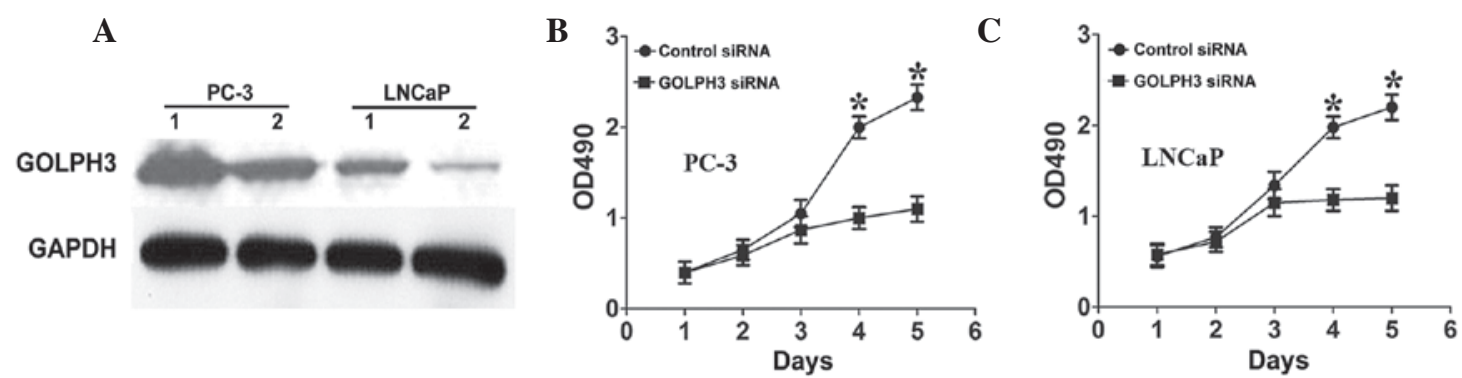

Figure 3. Effect of GOLPH3-siRNA on prostate cancer cell proliferation. (A) Western blot analysis indicated that GOLPH3-siRNA knocked down GOLPH3 expression. 1, Control-siRNA and 2, GOLPH3siRNA. (B) OD PC-3 and (C) LNCaP cells transfected with control siRNA or GOLPH3-siRNA was detected using MTT. Data are presented as the mean \pm standard deviation, ${ }^{*} \mathrm{P}<0.05$ vs. control siRNA-transfected cells. GOLPH3, golgi phosphoprotein 3; siRNA, small interfering RNA; OD, optical density.

$\mathbf{A}$
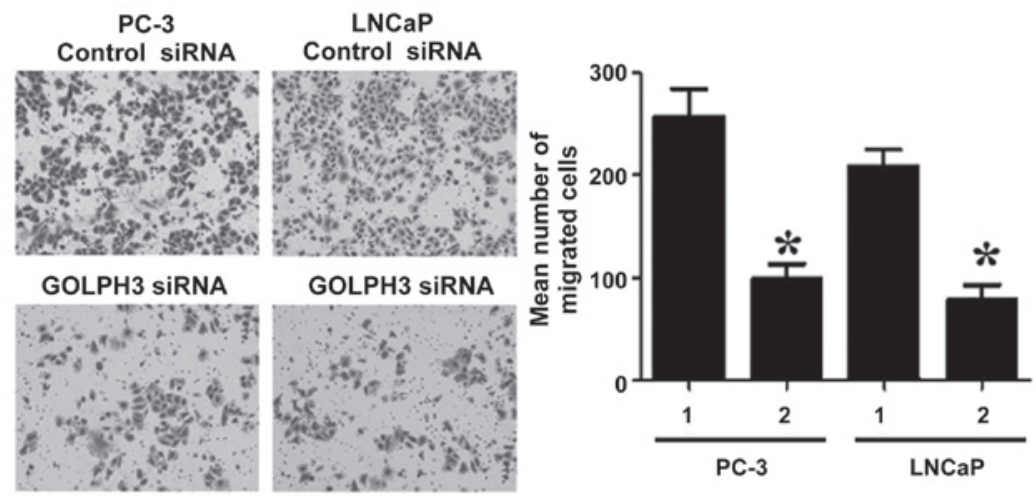

B
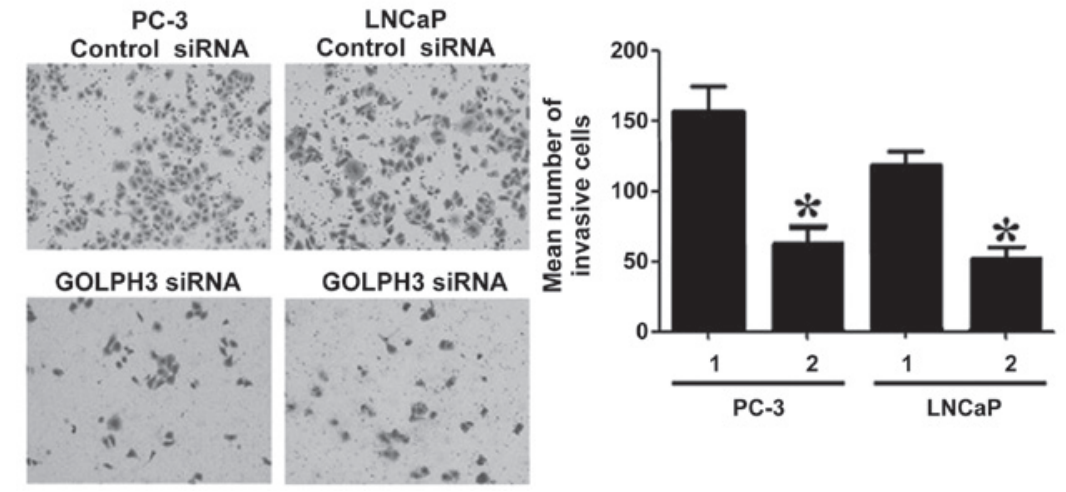

Figure 4. Prostate cancer cell migration and invasion. (A) Cells transfected with control siRNA or GOLPH3-siRNA for $48 \mathrm{~h}$ and, following a further $48 \mathrm{~h}$, migrated cells were stained and counted using a microscope (x10). Representative images are shown. (B) Cell invasion (x10). 1, control siRNA-transfected cells and 2, GOLPH3-siRNA-transfected cells. Data are shown as the mean \pm standard deviation from five fields. ${ }^{*} \mathrm{P}<0.05$ vs. control siRNA-transfected cells. GOLPH3, golgi phosphoprotein 3; siRNA, small interfering RNA. 
Silencing of GOLPH3 reduces proliferation, migration and invasion of PCa cells in vitro. Western blot analysis demonstrated that transfection with GOLPH3-siRNA knocked down GOLPH3 protein expression (Fig. 3A), and MTT assays suggested that the increase in PC-3 and LNCaP cell numbers over five days were significantly reduced following transfection with GOLPH3-siRNA (Fig. 3B and C). Cell migration assays (Fig. 4A) demonstrated that GOLPH3-siRNA transfection significantly reduced the migratory capability of PC-3 and LNCaP cell lines, compared with that in cells transfected with the control siRNA. Furthermore, cell invasion was significantly lower in GOLPH3-siRNA-transfected cell, compared with that in the control siRNA-transfected cells (Fig. 4B). These results indicated that GOLPH3 expression may induce PCa cell proliferation, migration and invasion.

\section{Discussion}

To the best of our knowledge, the association between GOLPH3 and PCa progression has yet to be investigated. In the present study, positive GOLPH3 expression was observed in $56 \%$ of PCa tissues compared with $6 \%$ of BPH tissues. In addition, positive GOLPH3 was significantly correlated with impaired biochemical recurrence-free survival and overall survival. Further analysis indicated that GOLPH3 expression is a potential independent factor indicating a poor prognosis in patients with $\mathrm{PCa}$.

The gene encoding GOLPH3 in humans is located on chromosome $5 \mathrm{p} 13$ and is expressed in several solid tumor types, including carcinoma of the lung, ovary, breast and skin (10). However, the association between GOLPH3 expression and PCa remains largely unknown. Recent studies have indicated that overexpression of GOLPH3 promotes tumorigenesis and progression in a number of types of malignancies, and is associated with poor survival in patients with cancer. GOLPH3 expression was shown to be present in $>50 \%$ of the 76 patients with glioma who were studied, and GOLPH3 expression levels were associated with the severity of the tumor, with higher GOLPH3 expression levels observed in higher grade astrocytomas (12). GOLPH3 overexpression is associated with poor prognosis in cN0 oral tongue cancer patients and may represent a novel and useful prognostic indicator for $\mathrm{cN} 0$ oral tongue cancer (17). The results of a study using cultured glioblastoma multiforme cell lines, suggested that the downregulation of GOLPH3, using an siRNA, led to the suppression of cell proliferation and clonogenic growth. These observations are in accordance with the findings of an association between high levels of GOLPH3 expression and a poor prognosis in patients with glioblastoma multiforme (18). Markedly higher levels of mRNA and protein GOLPH3 expression were observed in esophageal squamous cell cancer (ESCC) cell lines and tissues, compared with control cells (19). Expression of GOLPH3 in patients with ESCC was found to be positively associated with clinical stage, TNM classification and histological differentiation. Furthermore, expression of GOLPH3 is an independent prognostic factor for patients with ESCC (20). In gastric cancer, expression levels of GOLPH3 were found to be positively associated with tumor size, histological grade, depth of invasion, lymph node metastasis, distant metastasis and TNM stage. In a multivariate analysis the level of
GOLPH3 expression was an independent prognostic factor for patients with gastric cancer following radical resection (21). In accordance with these findings, the results of the present study suggested that increased positive GOLPH3 staining was observed in PCa tissues compared with that in non-PCa tissues. Furthermore, a positive GOLPH3 staining result was positively associated with Gleason score, T stage and lymph node status. Patients with positive GOLPH3 expression exhibited shorter biochemical recurrence-free, and overall survival. Furthermore, multivariate analyses suggested that GOLPH3 expression was an independent indicator of both biochemical recurrence-free and overall survival. Silencing of GOLPH3 expression inhibited the proliferation, migration and invasion capabilities of PC-3 and LNCaP cell lines.

In conclusion, the results of the present study suggested that GOLPH3 is involved in the proliferation, migration and invasion of PCa cells. Therefore, GOLPH3 may provide a novel prognostic marker for patients with $\mathrm{PCa}$ who have undergone radical prostatectomy.

\section{References}

1. Denmeade SR and Isaacs JT: Development of prostate cancer treatment: the good news. Prostate 58: 211-224, 2004.

2. Sarkar D, Lebedeva IV, Su ZZ, et al: Eradication of therapy-resistant human prostate tumors using a cancer terminator virus. Cancer Res 67: 5434-5442, 2007.

3. Siegel R, DeSantis C, Virgo K, Stein K, Mariotto A, Smith T, Cooper D, Gansler T, Lerro C, et al: Cancer treatment and survivorship statistics, 2012. CA Cancer J Clin 62: 220-241, 2012.

4. Hull GW, Rabbani F, Abbas F, Wheeler TM, Kattan MW and Scardino P: Cancer control with radical prostatectomy alone in 1,000 consecutive patients. J Urol 167: 528-534, 2002.

5. Patel AR and Stephenson AJ: Radiation therapy for prostate cancer after prostatectomy: adjuvant or salvage? Nat Rev Urol 8: 385-392, 2011

6. Mishra MV, Champ CE, Den RB, Scher ED, Shen X, Trabulsi EJ, Lallas CD, Knudsen KE, Dicker AP and Showalter TN: Postprostatectomy radiation therapy: an evidence-based review. Future Oncol 7: 1429-1440, 2011.

7. Chang AJ, Autio KA, Roach M III and Scher HI. High-risk prostate cancer-classification and therapy. Nat Rev Clin Oncol 11: 308-323, 2014.

8. Swanson GP and Basler JW: Prognostic factors for failure after prostatectomy. J Cancer 2: 1-19, 2010.

9. Crawford ED, Bennett CL, Andriole GL, Garnick MB and Petrylak DP: The utility of prostate-specific antigen in the management of advanced prostate cancer. BJU Int 112: 548-560, 2013.

10. Scott KL, Kabbarah O, Liang MC, Ivanova E, Anagnostou V, et al: GOLPH3 modulates mTOR signalling and rapamycin sensitivity in cancer. Nature 459: 1085-1090, 2009.

11. Kunigou O, Nagao H, Kawabata N, Ishidou Y, Nagano S, et al: Role of GOLPH3 and GOLPH3L in the proliferation of human rhabdomyosarcoma. Oncol Rep 26: 1337-1342, 2011.

12. Li XY, Liu W, Chen SF, Zhang LQ, Li XG and Wang LX: Expression of the Golgi phosphoprotein-3 gene in human gliomas: a pilot study. J Neurooncol 105: 159-163, 2011.

13. Romanuik TL, Wang G, Holt RA, Jones SJ, Marra MA and Sadar MD: Identification of novel androgen-responsive genes by sequencing of LongSAGE libraries. BMC Genomics 10: 476, 2009.

14. Abraham RT: GOLPH3 links the Golgi network to mTOR signaling and human cancer. Pigment Cell Melanoma Res 22: 378-379, 2009.

15. Zeng Z, Lin H, Zhao X, Liu G, Wang X, Xu R, Chen K, Li J and Song L: Overexpression of GOLPH3 promotes proliferation and tumorigenicity in breast cancer via suppression of the FOXO1 transcription factor. Clin Cancer Res 18: 4059-4069, 2012.

16. Steuber T, Erbersdobler A, Graefen M, Haese A, Huland H and Karakiewicz PI: Comparative assessment of the 1992 and 2002 pathologic T3 substages for the prediction of biochemical recurrence after radical prostatectomy. Cancer 106: 775-782, 2006. 
17. Li H, Guo L, Chen SW, Zhao XH, Zhuang SM, Wang LP, Song LB and Song M: GOLPH3 overexpression correlates with tumor progression and poor prognosis in patients with clinically N0 oral tongue cancer. J Transl Med 10: 168, 2012.

18. Zhou J, Xu T, Qin R, et al: Overexpression of Golgi phosphoprotein 3 (GOLPH3) in glioblastoma multiforme is associated with worse prognosis. J Neurooncol 110: 195-203, 2012.

19. Hua X, Yu L, Pan W, Huang X, Liao Z, Xian Q, Fang L and Shen H: Increased expression of Golgi phosphoprotein 3 is associated with tumor aggressiveness and poor prognosis of prostate cancer. Diagn Pathol 7: 127, 2012.
20. Wang JH, Chen XT, Wen ZS, Zheng M, Deng JM, Wang MZ, Lin HX, Chen K, Li J, et al: High expression of GOLPH3 in esophageal squamous cell carcinoma correlates with poor prognosis. PLoS One 7: e45622, 2012.

21. Hu BS, Hu H, Zhu CY, Gu YL and Li JP: Overexpression of GOLPH3 is associated with poor clinical outcome in gastric cancer. Tumor Biol 34: 515-520, 2013. 